\title{
TAON: A Topology-Oriented Active Overlay Network Protocol ${ }^{\star}$
}

\author{
Xinli Huang, Fanyuan Ma, and Wenju Zhang \\ Department of Computer Science and Engineering, \\ Shanghai Jiao Tong University, Shanghai, P.R. China, 200030 \\ \{huang-xl,ma-fy,zwj03\}@sjtu.edu.cn
}

\begin{abstract}
Built upon overlay topologies, Peer-to-Peer (P2P) networks behave in an ad-hoc way, conduct application-layer routing, enable usercustomized decentralized resources sharing, and thus can be taken as an emerging representative of Active Networks. An important problem in current unstructured P2P networks is that, however, existing searching mechanisms do not scale well because they are either based on the idea of flooding the network with queries or because they know very little about the nature of the network topology. In this paper, we propose the $\underline{T}$ Topology-oriented A Active $\underline{\text { Overlay }} \underline{\text { Network }}$ (TAON) which is an efficient, scalable yet simple protocol for improving decentralized resources sharing in P2P networks. TAON consists of three novel components: a Desirable Topology Construction and Adaptation algorithm to guide the evolution of the overlay topology towards a small-world-like graph, a Semanticbased Neighbor Selection Scheme to conduct an online neighbor ranking, and a Topology-aware Intelligent Search mechanism to forward incoming queries to deliberately selected neighbors. We deploy and compare TAON with a number of other distributed search techniques over static and dynamic environments, and the results indicate that TAON outperforms its competitors by achieving higher recall rate while using much less network resources, in both of the above environments.
\end{abstract}

\section{Introduction}

In contrast to traditional data networks, Active Networks not only allow the network nodes to perform computations on the data but also allow their users to inject customized programs into the nodes of the network, that may modify, store or redirect the user data flowing through the network [1]. These programmable networks open many new doors for possible applications that were unimaginable with traditional data networks. For example, Peer-to-Peer (P2P) overlay networks conduct application-layer routing in an ad-hoc way, enable user-customized decentralized resources sharing, and thus can be taken as an emerging representative of Active Networks.

\footnotetext{
* This research work is supported in part by the the National High Technology Research and Development Program of China (863 Program), under Grant No. 2004AA104270.
}

D. Hutchison et al. (Eds.): IWAN 2005, LNCS 4388, pp. 247-252, 2009.

(C) IFIP International Federation for Information Processing 2009 
The most dominate application in use on current P2P networks is large-scale distributed file sharing, especially the Web-based search applications. YouSearch 2] maintains a centralized search registry for query routing, making it difficult to adapt the search process to the heterogeneous and dynamic contexts of the peer users. A more distributed approach is to completely decentralize search, as in Gnutella 3. Queries are sent and forwarded blindly by each peer. But since the peer network topology is uncorrelated with the interests of the peer users, peers are flooded by requests and cannot effectively manage the ensuing traffic. Adaptive, content based routing has been proposed to overcome this difficulty in the file sharing setting. NeuroGrid 4 employs a learning mechanism to adjust metadata describing the contents of nodes. A similar idea has been proposed to distribute and personalize Web search using a query-based model and collaborative filtering [5. Search however is disjoint from crawling, making it necessary to rely on centralized search engines for content. The major limitation of these systems lies in their relatively poor search performance and ignorance of the nature of the underlying topology, which results in fatal scaling problem.

To address the scalability limitations mentioned above, we in this paper consider the Web information retrieval problem and propose a Topology-oriented Active Overlay Network ( $T A O N)$ protocol. TAON allows for symbiotic interactions of Web crawling and searching, whereby a peer can vertically adapt to its users's search interests, while horizontally peers can achieve better coverage by learning to collaboratively route and respond to queries.

TAON consists of three key components: a Desirable Topology Construction and Adaptation algorithm to guide the evolution of the overlay topology towards a power-law graph, a Semantic-based Neighbor Selection scheme to conduct an online neighbor ranking, and a Topology-Aware Intelligent Search mechanism to forward incoming queries to deliberately selected neighbors. We predict that the resultant topology for such a network is a small world, allowing for any two peers to reach each other via a short path (small diameter) while maximizing the efficiency of communications within clustered peer communities. To evaluate the performance gains of TAON, we will deploy and compare TAON with a number of other distributed search techniques over static and dynamic environments, through extensive simulations.

The rest of this paper is organized as follows: In Section 2, we detail the design of TAON. In Section 3 and 4, we present the experimental setup and the simulation results respectively. We conclude this paper in the last section.

\section{TAON Design}

The objective of TAON is to help the querying peer to find the most relevant answers to its query quickly and efficiently rather than finding the largest number of answers. To achieve this goal, TAON exploits both the semantic and geographical locality to construct small-world-like peer communities, by incorporating three novel techniques presented below respectively, in a very brief fashion due to the space limitation. 


\subsection{Desirable Topology Construction and Adaptation}

The Desirable Topology Construction and Adaptation algorithm is the core component that connects the TAON node to the rest of the network. To obtain desirable overlay topology and sequentially adapt it towards a better one dynamically, we prefer to keeping the out-degree of the network following a powerlaw distribution, and expect that this unique topological property, together with the other two novel techniques (to be addressed in the Section 2.2 and 2.3), will produce the desired "small-world" phenomena 6 .

We achieve the goal by adding and deleting links in a way that the total number of outgoing links at each node is conserved. We choose a node $A$ at random, build a link from this node to a new node $B$ chosen by a certain metric, and then immediately delete an existing link say with $C$ to conserve links at $A$. In addition, by increasing the fraction of links rewired we get the required short path length. If the fraction of links deleted and rewired is $p$, then for very small $p$ the average path length $L(p)$ comes down by orders of magnitude and is close to that of a random graph whereas the clustering coefficient $C(p)$ is still much large similar to that of a regular graph [7].

\subsection{Semantic-Based Neighbor Selection}

To decide to which peers a queries will be sent, a node ranks all its neighbors with respect to a given query. To do this, each node maintains a profile for each of its neighbor peers. The profile contains the list of the most recent past queries, which peers provided an answer for a particular query as well as the number of results that a particular peer returned. According to [8], we use the Cosine Similarity function below to compute the similarity between different queries:

$$
\operatorname{sim}\left(q, q_{i}\right)=\cos \left(q, q_{i}\right)=\frac{\sum\left(\boldsymbol{q} * \boldsymbol{q}_{\boldsymbol{i}}\right)}{\sqrt{\sum(\boldsymbol{q})^{2}} * \sqrt{\sum\left(\boldsymbol{q}_{\boldsymbol{i}}\right)^{2}}},
$$

where $\operatorname{sim}\left(q, q_{i}\right)$ is the similarity of the query $q$ and the query $q_{i}$, calculated by the cosine of the angle between the two vectors $\boldsymbol{q}$ and $\boldsymbol{q}_{\boldsymbol{i}}$.

Based on this similarity between queries, we then use the relevance function in 9 ] to rank the neighbor peers of a node $P_{0}$ following:

$$
R_{P_{0}}\left(P_{i}, q\right)=\sum_{j} \operatorname{sim}\left(q_{j}, q\right)^{\alpha} * S\left(P_{i}, q_{j}\right),
$$

where $\alpha$ is a parameter allowing us to add more weight to the most similar queries, $j$ is the ID of each query answered by $P_{i}, S\left(P_{i}, q_{j}\right)$ is the number of results returned by $P_{i}$ for query $q_{j}, R_{P_{0}}\left(P_{i}, q\right)$ denotes the relevance rank function of $P_{i}$ and is used by $P_{0}$ to perform an online ranking of its neighbors.

The $R$ function allows us to rank higher the peers that returned more relevant results, and thus realizes the semantic-based neighborhood optimization. In addition, we also make this semantic-based better neighbor selection strategy be orthogonal to the physical proximity based strategy that is integrated into the Topology-aware Intelligent Search mechanism (Section 2.3). 


\subsection{Topology-Aware Intelligent Search}

To make the TAON node in the overlay topology being aware of the physical proximity in the underlying network, we divide its neighbors into local neighbors and global neighbors. The fraction of neighbors that are local, called the proximity factor $(\alpha)$, is a key design parameter that governs the overall structure of the topology. Different values of $\alpha$ let us span the spectrum of this class of overlay topologies. In between these two ends of the spectrum, we foresee that the topologies, with many local links and a few global links, have desirable properties: They not only have low diameter, large search space and connectedness, but are also aware of the underlying network and can utilize these links better than the both topologies. We aim to find a suitable balance between these advantages by simulation through populating the range of $\alpha$ value.

The combination of the Semantic-based Neighbor Selection and the Physical Proximity based Neighbor Discrimination ensure that increasing queries from a node $P$ can be answered by its neighbor nodes or their nearby nodes in the overlay topology, and that many such answerers may be geographically close to the requester. These properties are especially useful for reducing response time and network resources consumption. Based on these techniques, we then develop a novel search mechanism, called Topology-aware Intelligent Search, to conduct a kind of bi-forked and directed search as follows:

- flooding the incoming queries to all local neighbors with a much smaller TTL value than that of the standard Gnutella protocol.

- forwarding the incoming queries to the first $k$ best global neighbors using multiple random walks coupled with the mechanisms of termination checking and duplication avoiding proposed in [10.

Here, all the local and global neighbors are beforehand selected and optimized using the Semantic-based Neighbor Selection strategy, and are discriminated by their physical proximity.

\section{Experimental Setup}

TAON is designed to perform efficient, scalable yet simple Web-based search by exploiting the locality semantically and geographically. Hence, our experimental evaluation focuses on four performance metrics below:

- recall rate, that is, the fraction of documents that the search mechanisms retrieves.

- search efficiency, that is, the number of messages used to find the results as well as the required time to locate the results.

- utilization of underlying network, measured by the traffic load on the links in the underlying network, according to [1].

- small-world statistics, as a indicator to show whether the network topology is being evolved towards a "small-world" graph, and measured using the factors of clustering coefficient and diameter. 
Based on the PLOD topology generator [12, we create a simulator (in which the TAON protocol is implemented and deployed) that initiates a power-law overlay topology and allows users to run their queries over real indexes obtained from actual distributed Web crawlers. Our simulator takes a snapshot of the network for every time step, during which all of the peers process all of their buffered incoming messages and send them following the TAON protocol. This may include the generation of a local query as well as forwarding and responding to the queries received by other peers.

\section{Simulation Results}

In this section, we describe a series of experiments that attempt to investigate the performance gains of TAON over its competitors of a) Breadth First Search (BFS, i.e., Gnutella), b) Random Walks (RW), and the Most Results in Past (MRP, i.e., the technique proposed in [13]).
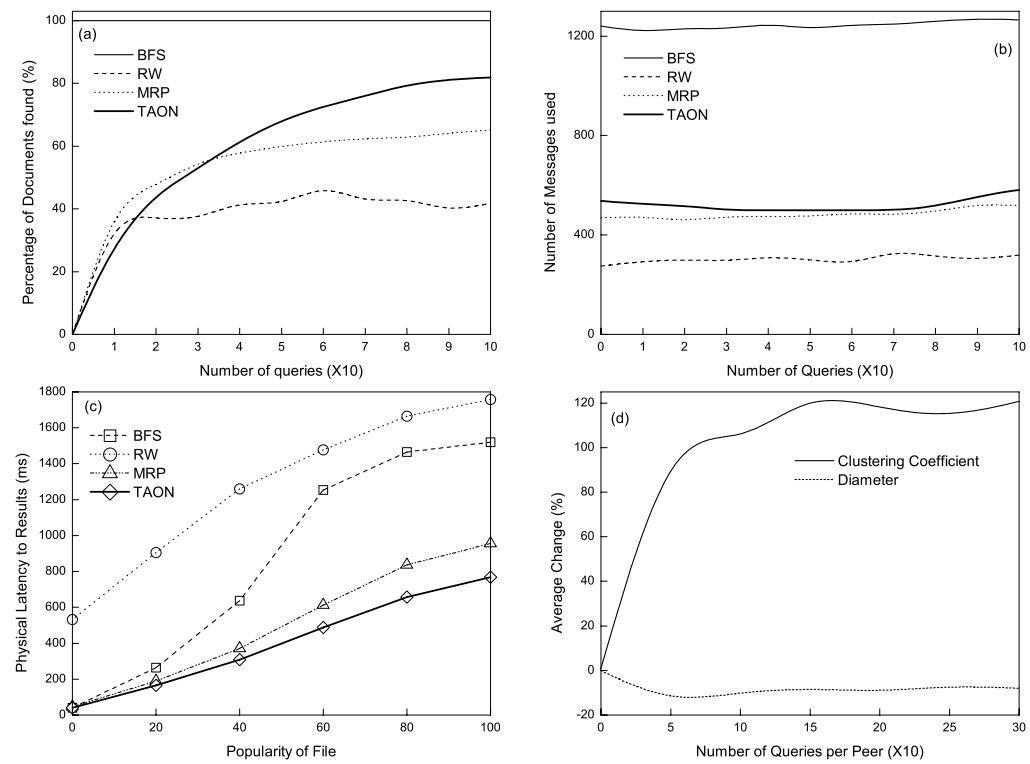

Fig. 1. Comparisons of Recall Rate (a), Messages (b), and Physical Latency to Results (c) between the four search techniques, and Small-World Statistics of the TAON network (d)

Fig I. (a) and (b) indicate that BFS requires almost three times as many messages as its competitors with around 1,230 messages per query. In contrast, all of RW, MRP and TAON use dramatically less messages but TAON is the one that finds the most documents. In addition, the curves in Fig (1) (c) shows clearly that TAON results in smaller physical latency than the other three techniques, which means a better utilization of the underlying physical network. 
Fig 1. (d) shows that the diameter remains roughly equal to the initial random graph diameter, while the clustering coefficient increases rapidly and significantly, stabilizing around a value $100 \sim 125 \%$ larger than that of the initial random graph. These conditions define the emergence of a "small world" topology in the TAON network. This is a very interesting finding, indicating that the peer interactions cause the peers to route queries in such a way that communities of users with similar interests cluster together to find qualified results quickly, while it is still possible to reach any peer in a small number of steps.

\section{Conclusions}

The TAON protocol we proposed in this paper provably results in significant performance gains of both enhanced search efficiency and reduced traffic load, by explicitly guaranteeing the desirable topological properties like small-world properties, and by exploiting the semantic and geographical locality to form better neighborhood and peer communities dynamically.

\section{References}

1. David, L., David Sincoskie, W., Wetherall, D.J., Minden, G.J.: A Survey of Active Network Research. IEEE Transactions on Communications (1997)

2. Bawa, M., et al.: Make it fresh, make it quick searching a network of personal webservers. In: Proc. of 12th WWW (2003)

3. http://rfc-gnutella.sourceforge.net

4. Joseph, S.: Neurogrid: Semantically routing queries in Peer-to-Peer networks. In: Proc. of Intl. Work. P2P Computing (2002)

5. Pujol, J., Sangüesa, R., Bermúdez, J.: Porqpine: A distributed and collaborative search engine. In: Proc. of 12th WWW (2003)

6. Watts, D., Strogatz, S.: Collective dynamics of 'small-world' networks. Nature 393, 440-442 (1998)

7. Puniyani, A.R., Lukose, R.M., Huberman, B.A.: Intentional Walks on Scale Free Small Worlds. LANL archive: cond-mat/0107212 (2001)

8. Baeza-Yates, R.A., Ribeiro-Neto, B.A.: Modern Information Retrieval. ACM Press Series/Addison Wesley, New York (1999)

9. Zeinalipour-Yazti, D., Kalogeraki, V., Gunopulos, D.: Exploiting locality for scalable information retrieval in peer-to-peer networks. Information Systems 30(4), 277-298 (2005)

10. Lv, C., et al.: Search and replication in unstructured peer-to-peer networks. In: Proc. of ACM International Conference on Supercomputing (ICS) (June 2002)

11. Ripeanu, M., Foster, I., Iamnitchi, A.: Mapping the Gnutella Network: Properties of Large Scale Peer-to-Peer Systems and Implications for System Design. IEEE J. on Internet Computing, Special Issue on Peer-to-peer Networking (2002)

12. Palmer, C.R., Steffan, J.G.: Generating Network Topologies That Obey Powers. In: Proc. of Globecom 2000, San Francisco (November 2000)

13. Yang, B., Garcia-Molina, H.: Efficient Search in Peer-to-Peer Networks. In: Proc. of Int. Conf. on Distributed Computing Systems (2002) 